Psychotherapeut 2022 $67: 501-508$ https://doi.org/10.1007/s00278-021-00541-2 Angenommen: 6. September 2021 Online publiziert: 27. Oktober 2021 (c) Der/die Autor(en) 2021

\title{
Kölner Fragebogen zur Sprachlosigkeit
}

\section{Empirische Prüfung eines Schwellenwerts für den klinischen Einsatz}

Thilo Dietz ${ }^{1}$. Vera Schiewer ${ }^{1}$ S Sally Tavenrath ${ }^{1} \cdot$ Hülya Öztürk-Arenz ${ }^{1}$ Anne Klein ${ }^{1}$. Hildegard Labouvie ${ }^{1} \cdot$ Reinhold Jäger ${ }^{2} \cdot$ Michael Kusch $^{1}$

'Psychoonkologische Versorgungsforschung, Klinik I für Innere Medizin, Universitätsklinikum Köln, Köln, Deutschland

${ }^{2}$ Zentrum für Empirische Pädagogische Forschung, Universität Koblenz-Landau, Campus Landau, Landau, Deutschland

\section{Zusammenfassung}

Hintergrund: Mit dem Kölner Fragebogen zur Sprachlosigkeit (KFS) liegt erstmals ein validiertes Erhebungsinstrument zur Erfassung von Sprachlosigkeit vor. Die empirische Prüfung des KFS hinsichtlich seiner Eignung zur validen Unterscheidung von Personen mit gering und hoch ausgeprägter Sprachlosigkeit ist von zentraler Bedeutung für den klinischen Einsatz des Instruments.

Material und Methoden: Die Stichprobe umfasste die KFS-Daten von insgesamt 205 teilnehmenden Personen einer Onlineerhebung. Mithilfe mehrerer inferenzstatistischer Verfahren wurden die prädiktiven Eigenschaften des KFS-Item 12 analysiert, um es für die Bestimmung eines Schwellenwerts beim Einsatz des KFS nutzbar zu machen. Basierend auf dem 75. Perzentil des KFS-Item 12 erfolgte eine Dichotomisierung der Stichprobe mit anschließender "Receiver-operating-characteristic"(ROC)-Analyse zur KFS-Gesamtskala.

Ergebnisse: Die Ergebnisse der ROC-Analyse („area under the curve“ [AUC]: 0,863; $p<0,001)$ ergaben bei Verwendung des Youden-Index den optimalen Schwellenwert von $>29$, mit einer Sensitivität von $76 \%$ und einer Spezifität von $79 \%$ für die Gesamtskala des KFS.

Diskussion: Der Schwellenwert der KFS-Gesamtskala ist zur Differenzierung von Personen mit gering oder stark ausgeprägter Sprachlosigkeit geeignet. Das Item 12 des KFS kann zudem als Prädiktor einer potenziellen Sprachlosigkeit herangezogen werden.

\section{Schlüsselwörter}

Screening $\cdot$ Schwellenwert $\cdot$ ROC-Kurve $\cdot$ Sensitivität und Spezifität · Affektive Symptome

Mit dem Kölner Fragebogen zur Sprachlosigkeit (KFS) (Dietz et al. 2021) liegt der erste, validierte Fragebogen zur Erfassung des Konstrukts der Sprachlosigkeit vor. Um den Fragebogen in der klinischen Praxis zur Identifikation von Personen mit stark ausgeprägter Sprachlosigkeit einsetzen zu können, bedarf es der Bestimmung eines gesicherten Schwellenwerts. Ziel der vorliegenden Untersuchung ist diese empirische Validierung.

\section{Grundlagen}

Schwellenwerte zur psychometrisch gestützten Differenzialdiagnostik

Psychologische Testverfahren sind vor ihrer klinischen Anwendung einer psychometrischen Prüfung zu unterziehen (Kubinger 2003). Werden die entsprechenden Vorgaben nachgewiesen, ist das Instrument für einen Einsatz in der klinischen Praxis akzeptabel. 
Aus klinischer Perspektive erfolgt die Nutzung psychologischer Testverfahren zur psychometrisch gestützten Differenzialdiagnostik, d.h. zur Differenzierung der Ausprägungsgrade der sich in Behandlung befindlichen Personen; dies ermöglicht eine zielgerichtete Intervention (Eskildsen et al. 2020; Kusch und Stecker 2005; Kusch et al. 2013, 2015; Mazzocco Myers 2003). Zur Differenzierung ist die Verwendung von Schwellenwerten erforderlich, die eine entsprechende Klassifikation erlauben (Habibzadeh et al. 2016; Yovanoff und Squires 2006) und es ermöglichen, Personen eine entsprechende Intervention zukommen zulassen (Jäger und Sebastian 2010). Ergänzend gewinnen Schwellenwerte als Gegenstand komplexer Versorgungsprozesse an Bedeutung (Eskildsen et al. 2020; Kusch et al. 2015).

Die Bestimmung eines spezifischen Schwellenwerts gelingt mithilfe unterschiedlicher Verfahren (Goldhammer und Hartig 2020). Die normorientierte Testwertinterpretation betrachtet den individuellen Wert einer Person zu dessen Bezugsgruppe. Über die Transformation des Testwerts in einen Prozentrang steht das Antwortverhalten von Personen in Relation zum maximal erreichbaren Gesamtscore des Erhebungsinstruments (Goldhammer und Hartig 2020). So können beispielsweise Personen, die einen Wert größer als der Prozentrang von $75 \%$ ihrer Bezugsgruppe vorweisen, als behandlungsbedürftig definiert werden.

Eine Alternative zur normorientierten Testwertinterpretation bildet die kriteriumorientierte Schwellenwertbildung. Über ein externes Kriterium und die zugrunde liegende Theorie des Testverfahrens kann der optimale Schwellenwert mithilfe der Methode der "Receiver-operating-characteristic"(ROC)-Analyse ermittelt werden (Habibzadeh et al. 2016; Yovanoff und Squires 2006). Die Verwendung der ROC-Analyse bedarf jedoch eines bereits validierten Erhebungsinstruments mit (identischer) theoretischer Grundlage sowie des Vorhandenseins des Kriteriums seitens der zu untersuchenden Person (Goldhammer und Hartig 2020). Die Identifikation des optimalen Schwellenwerts mithilfe der ROC-Analyse kann methodisch auf unterschiedliche Vorgehensweisen erfolgen (Habibzadeh et al. 2016), wobei der
Youden-Index (Youden 1950) empfohlen wird (Perkins und Schisterman 2006). Der Youden-Index bildet sich aus der Addition von Sensitivität und Spezifität, subtrahiert um den Wert 1 (Youden 1950). Je größer der Index, desto besser ist die Trennung beider Gruppen durch den gegebenen Schwellenwert (Perkins und Schisterman 2006). Diese Vorgehensweise setzt jedoch a) eine umfangreiche Stichprobe zur Schwellenwertbildung oder b) ein bereits validiertes Erhebungsinstrument mit identischer theoretischer Grundlage zur Klassifikation von Personen(-gruppen) voraus.

\section{Phänomen der Sprachlosigkeit}

Primär beschreibt der Begriff Sprachlosigkeit einen vorübergehenden Zustand, der durch stark negative und positive emotionale Situationen ausgelöst wird (Berger et al. 2016; Dietz et al. 2021). Darüber hinaus sollen mit dem Begriff der Sprachlosigkeit auch zeitstabile Eigenschaften von Personen beschrieben werden, die nicht in der Lage sind, Worte für ihre Emotionen zu finden, da sie diese nicht wahrnehmen und/oder ihren verbalen Ausdruck unterbinden (Dietz et al. 2021; Schiewer et al. 2021). Im beobachtbaren Verhalten zeigt sich die Sprachlosigkeit als eine Situation des Schweigens in der Gesprächsführung (Berger et al. 2016). Schweigen kann zu einem Kreislauf zunehmender Verunsicherung (Canzona et al. 2019) und wachsender Isolation (Atkins et al. 2013; Konradsen et al. 2012) führen und mit psychischen Störungen einhergehen (Paoletti und BenSoussan 2020).

\section{Ziel der Arbeit}

Der KFS (Dietz et al., 2021) stellt das erste validierte Erhebungsinstrument zur Erfassung von Sprachlosigkeit dar. Bislang erfolgte noch keine Schwellenwertbildung; diese scheint vor dem Hintergrund der Konsequenz von Sprachlosigkeit, insbesondere für den klinischen Einsatz, empfehlenswert. Die Entwicklung und empirische Prüfung eines KFS-Schwellenwerts bildet den Gegenstand der vorliegenden Arbeit.

\section{Methode}

\section{Stichprobe}

Die Stichprobe umfasst 311 Personen. Diese wurde im Rahmen einer Onlineumfrage zur Sprachlosigkeit erfasst und stimmt mit der Stichprobe zur Validierung des KFS (Dietz et al. 2021) überein. Teilnehmende waren Mitarbeiterinnen und -arbeiter der Uniklinik Köln, die über einen Intranetbeitrag zur Teilnahme gebeten wurden. Zusätzlich wurden Studierende per E-MailVerteiler kontaktiert und eingeladen, an der Umfrage teilzunehmen.

Insgesamt 106 Personen (34\%) wurden aufgrund fehlender Angaben ausgeschlossen; der final ausgewertete Datensatz umfasste 205 Teilnehmende (66\%). Das Durchschnittalter betrug 26,63 Jahre ( $\hat{=}$ Mittelwert $[\mathrm{M}]$; Standardabweichung [SD] $\pm 12,74$ Jahre); es sind 172 Teilnehmende $(83,9 \%)$ weiblich. Mehr als die Hälfte der befragten Personen (54,7\%) gaben einen Hochschulabschluss (Promotion: 15,6\%; Diplom: 8,3\%; Master: 17,6\%; Bachelor: 13,2\%) an, 14,6\% einen Lehrabschluss, $18 \%$ die allgemeine Hochschulreife und $12,7 \%$ einen anderen Bildungsabschluss. Angaben zu physischen oder psychischen Erkrankungen wurden im Rahmen der Umfrage nicht erfasst.

\section{Material}

\section{Kölner Fragebogen zur \\ Sprachlosigkeit}

Der KFS (Dietz et al. 2021) umfasst insgesamt 19 Items mit Antwortmöglichkeiten auf einer 6-stufigen Likert-Skala (0: „trifft überhaupt nicht zu“ bis 5: „trifft voll zu“). Inhaltlich gliedern sich die Items in vier Subskalen (1) Allgemeine Gefühlsbeschreibung, (2) Gefühlsbeachtung, (3) Gefühlsunsicherheit und (4) Positive Gefühlsbeschreibung. Zusätzlich wird die übergreifende Gesamtskala Sprachlosigkeit zur Erfassung einer komponentenübergreifenden Sprachlosigkeit, bestehend aus allen 19 Items, gebildet (Dietz et al. 2021).

\section{Indikatorvariable Item 12}

Die Konstruktion des KFS-Items 12 (Dietz et al.; „Andere sagen, ich soll mehr über meine Gefühle sprechen.") erfolgte in semantischer Anlehnung an Item 12 der deutsch- 


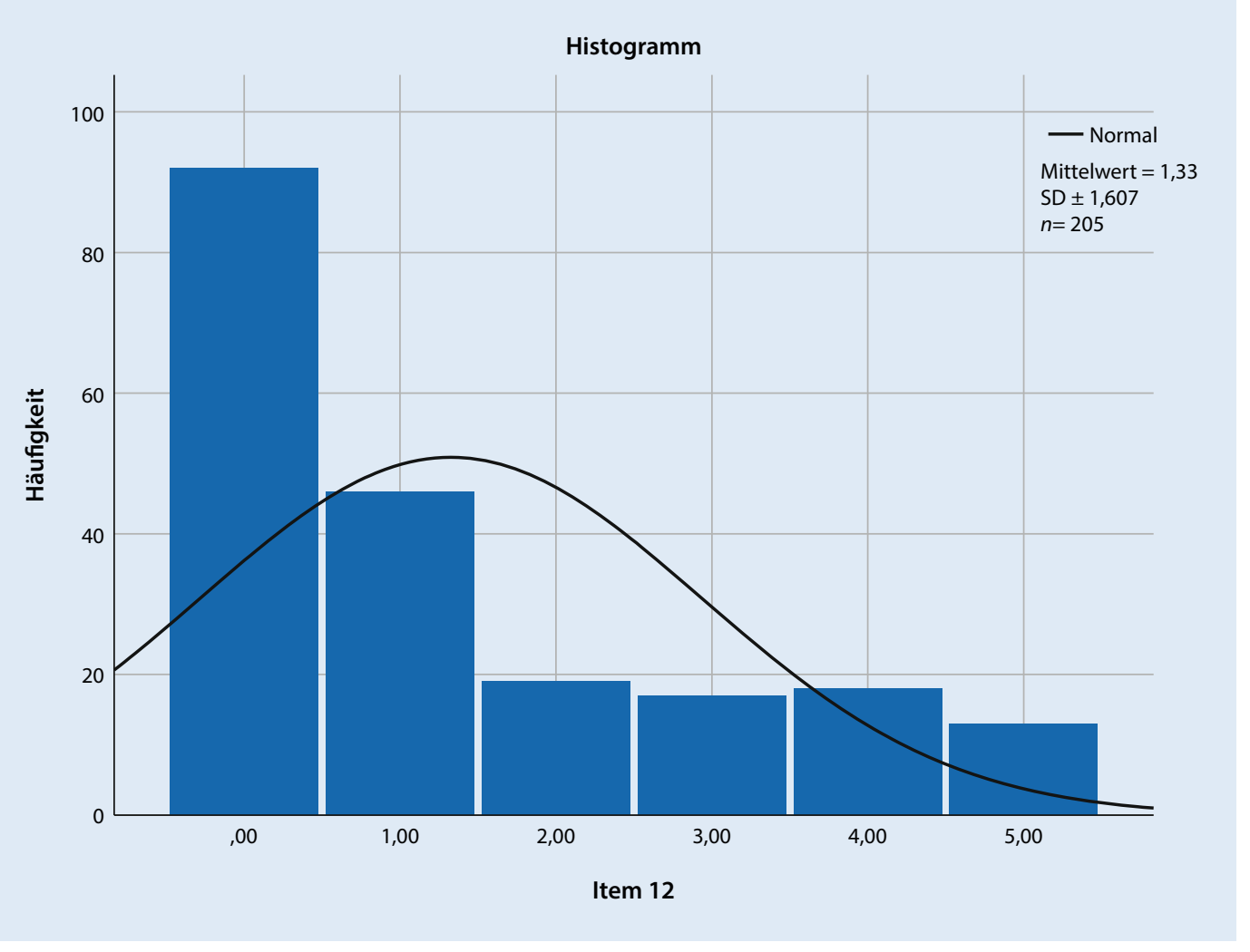

Abb. 1 ४ Verteilung der Rohwerte des Items 12 aus dem Kölner Fragebogen zur Sprachlosigkeit sprachigen Version der Toronto Alexithymia Scale-26 (TAS-26; Kupfer et al. 2001). Während Item 12 der TAS-26 (Kupfer et al. 2001; „Andere sagen, ich soll meine Gefühle mehr zeigen.") den Ausdruck von Emotionen erhebt, zielt die Formulierung des KFS-Item 12 (Dietz et al. 2021) explizit auf den sprachlichen Ausdruck der eigenen Gefühle ab. Item 12 des KFS (Dietz et al. 2021) kann als Indikatorvariable für gering bzw. stark ausgeprägte Sprachlosigkeit herangezogen und unter Gesichtspunkten der normorientierten Testwertinterpretation analysiert werden. Dies ist darin begründet, dass entsprechend den Annahmen der Item Response Theory (IRT; Moosbrugger et al. 2020) von einem bestehenden Zusammenhang zwischen latenten Merkmalsausprägungen und einem positiven Antwortverhalten auf Item-Ebene ausgegangen wird. Im Rahmen der Validierung des KFS (Dietz et al. 2021) erzielte Item 12 eine Itemschwierigkeit von $31 \%$ und eine Trennschärfe von 0,6. Diese Werte kennzeichnen das Item als optimale Ausgangslage für eine Schwellenwertbestimmung (Kelava und Moosbrugger 2020). Da es sich bei der Sprachlosigkeit um ein neues Konstrukt handelt, liegen zurzeit nur wenige Informationen über die Verteilung in der Allgemeinbevölkerung oder in spezifischen Personengruppen vor (Dietz et al. 2021). Es ist davon auszugehen, dass Personen mit einem Antwortverhalten (ItemRohwert) im letzten Quartil (>75. Perzentil) von Item 12 sprachloser erscheinen als vergleichbare Personen unter dem entsprechenden Wert. Unter Gesichtspunkten der IRT scheint es ungeeignet, Personen bereits bei einem Überschreiten des zweiten Quartils (>50. Perzentil) als auffällig zu identifizieren. Zusätzlich führt die Literatur (Goldhammer und Hartig 2020) an, dass insbesondere bei einer fehlenden Normalverteilung die Prozentränge zur Identifikation zu nutzen sind.

Item 12 eignet sich besonders, da dieses die Reflexion der eigenen gefühlsbezogen, verbalen Expression bedingt und entsprechend als externes Kriterium zur kriteriumorientierten Schwellenwertbildung herangezogen werden kann. In der Literatur finden sich verschiedene Beispiele für einzelne Items spezifischer Fragebogen unter Verwendung als "single items" mit guter Vorhersagekraft (Bergkvist und Rossiter 2007; Mitchell et al. 2012).

\section{Statistische Analyse}

Die Analysen erfolgten mithilfe des Statistikprogramms IBM SPSS Version 26. Entsprechend der theoretischen Annahme der IRT und der Eignung von Item 12 des KFS (Dietz et al. 2021) erfolgte die Identifikation des Prozentrang 75 als Schwellenwert zur Identifikation einer potenziell stark ausgeprägten Sprachlosigkeit mithilfe deskriptiv statistischer Analysen. Die Eignung des Items als Indikator für Sprachlosigkeit wurde mithilfe von Pearson-Produkt-Moment-Korrelationen und Regressionsanalysen unter Anwendung der Subund Gesamtskala des KFS (Dietz et al. 2021) nachgewiesen. Im Kontext der Schwellenwertbestimmung wurde Item 12 bei der Bildung der Skalenwerte der Subskala Gefühlsbeschreibung und der Gesamtskala Sprachlosigkeit nicht berücksichtigt, um verfälschende Ergebnisse auszuschließen. Final wurde eine ROC-Analyse zur Identifikation eines Schwellenwerts der Sprachlosigkeits-Gesamtskala, basierend auf der zuvor definierten Unterteilung über den Schwellenwert des KFS-Item 12 (Dietz et al. 2021), durchgeführt. Die Berechnung des optimalen Schwellenwerts erfolgte unter 


\section{Originalien}

Tab. 1 Regressionsgleichungen mit Item 12 als unabhängige Variable

\begin{tabular}{|c|c|c|c|c|c|c|c|}
\hline \multirow[t]{2}{*}{ AV } & \multicolumn{2}{|c|}{ Regressionskoeffizienten } & \multirow[t]{2}{*}{$\mathrm{T}$ (Item 12) } & \multirow[t]{2}{*}{$p$} & \multirow[t]{2}{*}{ Modellgüte $\mathrm{R}^{2}$} & \multicolumn{2}{|c|}{$95 \%$-Konfidenzintervall (Item 12) } \\
\hline & Konstante & Item 12 & & & & Untergrenze & Obergrenze \\
\hline AG (mod.) & 6,661 & 2,579 & 10,584 & $<0,001$ & 0,352 & 2,099 & 3,060 \\
\hline GB & 8,427 & 1,601 & 6,583 & $<0,001$ & 0,172 & 1,122 & 2,081 \\
\hline GU & 2,981 & 0,757 & 5,861 & $<0,001$ & 0,140 & 0,502 & 1,012 \\
\hline PG & 1,602 & 0,826 & 6,587 & $<0,001$ & 0,172 & 0,579 & 1,073 \\
\hline SL (mod.) & 19,670 & 5,763 & 10,454 & $<0,001$ & 0,347 & 4,676 & 6,850 \\
\hline
\end{tabular}

$n=205$
AV abhängige Variable, AG Allgemeine Gefühlsbeschreibung, GB Gefühlsbeachtung, GU Gefühlsunsicherheit, PG Positive Gefühlsbeschreibung, SL Sprachlosigkeit, mod. modifiziert

\begin{tabular}{|c|c|c|c|c|c|c|}
\hline \multirow[t]{3}{*}{ Skala } & \multicolumn{4}{|c|}{ Sprachlosigkeit } & \multirow{2}{*}{\multicolumn{2}{|c|}{ Gruppenunterschied }} \\
\hline & \multicolumn{2}{|c|}{$\begin{array}{l}\text { Gering ausgeprägt } \\
(n=138)\end{array}$} & \multicolumn{2}{|c|}{$\begin{array}{l}\text { Stark ausgeprägt } \\
(n=67)\end{array}$} & & \\
\hline & M & \pm SD & M & \pm SD & $T$ & $p$ \\
\hline AG & 7,61 & 5,33 & 19,24 & 6,87 & $-12,191$ & $<0,001$ \\
\hline GB & 8,92 & 5,56 & 13,91 & 5,94 & $-5,896$ & $<0,001$ \\
\hline GU & 3,17 & 2,64 & 5,66 & 3,59 & $-5,035$ & $<0,001$ \\
\hline PG & 1,81 & 2,45 & 4,52 & 3,66 & $-5,491$ & $<0,001$ \\
\hline $\mathrm{SL}$ & 21,51 & 11,56 & 43,33 & 15,99 & $-9,974$ & $<0,001$ \\
\hline
\end{tabular}

Tab. 3 Parameter der "Receiver-operating-characteristic“-Kurve unter Verwendung der Indikatorvariable von Item 12 des Kölner Fragebogen zur Sprachlosigkeit

\begin{tabular}{|c|c|c|c|c|c|c|c|c|}
\hline SW & SE (\%) & SP (\%) & J & $\mathrm{LR}^{+}$ & $\mathbf{L R}^{-}$ & AUC & $p$ & $95 \%-K I$ \\
\hline & & & & & & 0,863 & $<0,001$ & $0,812-0,914$ \\
\hline 27 & 82 & 70 & 0,524 & 2,73 & 0,26 & - & - & - \\
\hline 28 & 86 & 73 & 0,493 & 3,19 & 0,19 & - & - & - \\
\hline 29 & 76 & 79 & 0,551 & 3,62 & 0,30 & - & - & - \\
\hline 31 & 73 & 81 & 0,543 & 3,84 & 0,33 & - & - & - \\
\hline 32 & 72 & 83 & 0,550 & 4,24 & 0,34 & - & - & - \\
\hline
\end{tabular}

der Anwendung der Formel nach Youden (1950):

\section{$\mathrm{J}=$ Sensitivität + Spezifität -1}

Zudem wurden Diskriminanzanalysen zur Prüfung des Schwellenwerts herangezogen. Mittelwertunterschiede wurden mithilfe von $t$-Tests für unabhängige Stichproben berechnet.

\section{Ergebnisse}

Rohwertverteilung und Schwellenwertbildung von Item 12

Die Ergebnisse der deskriptiven Statistik zu Item 12 des KFS (Dietz et al. 2021) ergaben einen Modalwert von 0 und einen durchschnittlichen Item-Rohwert von $\mathrm{M}=1,33$ (SD $\pm 1,61$; Spannweite: $0-5$ ). Die Schiefe des Items weist auf eine linkssteile (Schiefe: 1,021) und flache Verteilung (Kurtosis: $-0,240$ ) hin (- Abb. 1).
Der Prozentrang 75 geht mit einem Rohwert von 2 einher. Insgesamt 138 Teilnehmende $(67,3 \%)$ erzielten einen Rohwert $<2,19(9,3 \%)$ einen Rohwert von 2 und $48(23,4 \%)$ einen Rohwert $>2$.

Die Korrelationsergebnisse wiesen auf einen signifikanten Zusammenhang zwischen Item 12 und den Subskalen Allgemeine Gefühlsbeschreibung (modifiziert unter Außer-Acht-Lassen von Item 12; $r=0,596 ; \quad p<0,001)$, Gefühlsbeachtung $(r=0,419 ; p<0,001)$, Gefühlsunsicherheit $(r=0,380 ; p<0,001)$, Positive Gefühlsbeschreibung $(r=0,420 ; p<0,001)$ und der 


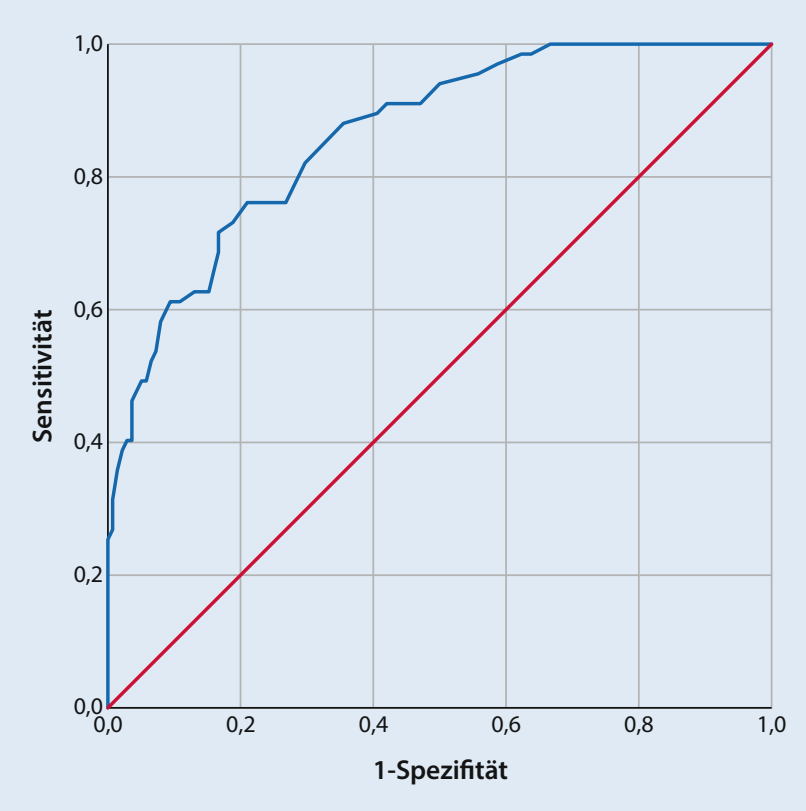

Abb. $2 \triangleleft$ „Receiveroperating-characteristic"-Kurve unter Verwendung der Indikatorvariable von Item 12 des Kölner Fragebogen zur Sprachlosigkeit
Sprachlosigkeits-Gesamtskala (modifiziert unter Außer-Acht-Lassen von Item 12; $r=0,592 ; p<0,001$ ) des KFS (Dietz et al. 2021) hin.

Unter Verwendung von Item 12 als unabhängige Variable erfolgte die Untersuchung des Einflusses des Items auf die Ausprägung der (Sub-)Skalenwerte des KFS (Dietz et al. 2021). Alle Regressionsanalysen wiesen signifikante Ergebnisse und eine hohe Modellgüte auf (Cohen 1988; - Tab. 3). Insbesondere für die modifizierte Sprachlosigkeit-Gesamtskala erwies sich Item 12 als geeigneter und kontinuierlicher Prädiktor mit hoher Evidenz (• Tab. 1).

Auf Basis der Ergebnisse wurde ein Schwellenwert für Item 12 gebildet. Alle Personen mit einem Rohwert $\geq 2$ (Prozentrang 75) wurden als Teilnehmende mit stark ausgeprägter Sprachlosigkeit klassifiziert. Die Mittelwerte beider Gruppen unterschieden sich für die Subskalen Gefühlsunsicherheit, Gefühlsbeschreibung und Gefühlsfokussierung sowie die Sprachlosigkeits-Gesamtskala signifikant voneinander (- Tab. 2). Personen mit einem Rohwert <2 erzielten einen Mittelwert von 21,51 (SD $\pm 11,56)$ und Teilnehmende der Gruppe mit stark ausgeprägter Sprachlosigkeit einen Mittelwert von 43,33 (SD $\pm 15,99)$. Ein $t$-Test für unabhängige Stichproben erwies sich für alle Skalen als signifikant. Insbesondere die Mittelwertdifferenz der Sprachlosigkeits-
Gesamtskala bestätigte die Verwendung des Item 12 als externes Kriterium zur Schwellenwertermittlung (• Tab. 2).

\section{Ermittlung des Schwellenwerts}

Auf Basis des Prozentrangs 75 (Rohwert $\geq 2$ ) des KFS-Items 12 (Dietz et al. 2021) als externes Kriterium erfolgte im Anschluss die Berechnung einer ROC-Analyse. Die „area under the curve" (AUC) erzielte mit einem Wert von 0,863 exzellente Ergebnisse (Backhaus et al. 2018; - Tab. 3; • Abb. 2). Unter Nutzung des Youden-Index (Youden 1950; J = 0,551) wurde ein KFS-Schwellenwert von >29 mit einer Sensitivität von $76 \%$ und einer Spezifität von $79 \%$ für die Sprachlosigkeits-Gesamtskala berechnet. Die Wahrscheinlichkeit, ein positives Testergebnis bei einer Person mit stark ausgeprägter Sprachlosigkeit zu finden (positive Likelihood-Ratio; $\mathrm{LR}^{+}$), betrug 3,62.

\section{Diskriminanzanalyse}

Die Personen wurden entsprechend dem KFS-Schwellenwert $>29$ in unterschwellig sprachlos und überschwellig sprachlos gruppiert. Die 4 Subskalen des KFS (Dietz et al. 2021) sowie die Gesamtskala wurden als unabhängige Variablen in eine unabhängige Diskriminanzanalyse einbezogen. Die Diskriminanzfunktion erreichte ein signifikantes Ergebnis $(p<0,001)$, bei einem
Eigenwert von 2,115 . Wilks $\lambda$ betrug 0,321 $(p<0,001)$ sowie die Gruppenzentroiden $-1,158$ für unterschwellig sprachlose und 1,809 für überschwellig sprachlose Personen. Die Subskala Positive Gefühlsbeschreibung erfüllte die Bedingungen des Toleranztests nicht; die übrigen Subskalen Allgemeine Gefühlsbeschreibung, Gefühlsbeachtung und Gefühlsunsicherheit erzielten einen standardisierten kanonischen Diskriminanzkoeffizienten von 0,058 (Allgemeine Gefühlsbeschreibung), 0,197 (Gefühlsbeachtung) bzw. 0,154 (Gefühlsunsicherheit). Die SprachlosigkeitsGesamtskala erzielte einen Koeffizienten von 0,766 . Insgesamt $96,6 \%$ der Fälle konnten unter Hinzunahme aller KFS(Sub-)Skalen korrekt klassifiziert werden. Ergänzend wurde die Diskriminanzanalyse mit der Sprachlosigkeits-Gesamtskala als alleinige unabhängige Variable wiederholt. Wilks $\lambda$ erzielte einen höheren Wert $(\lambda=0,325 ; p<0,001)$ gegenüber der vorausgegangenen Analyse. Die Gruppenzentroiden für unterschwellig sprachlose Personen betrugen -1.147 (• Abb. 3) und für überschwellig sprachlose Teilnehmende 1,792 (• Abb. 4). Insgesamt konnten 95,6\% (vgl. Diskriminanzanalyse mit allen KFS-[Sub-]Skalen: 96,6\%) der Fälle korrekt klassifiziert werden.

\section{Diskussion}

Ziel der vorliegenden Arbeit war die empirische Bestimmung eines Schwellenwerts für den KFS (Dietz et al. 2021). Bedingt durch das noch neue Konstrukt der Sprachlosigkeit liegen bisher keine weiteren Erhebungsinstrumente vor, anhand derer sich ein solcher Schwellenwert empirisch bestimmen ließe. Entsprechend erfolgte die Verwendung eines testeigenen Items zu Differenzierung und Klassifizierung der Personen in gering bzw. stark ausgeprägte Sprachlosigkeit.

Die Studie weist mit 83,9\% einen hohen Anteil weiblicher Teilnehmerinnen auf. Im Rahmen der Validierung des KFS (Dietz et al. 2021) wurde bereits deutlich, dass keine geschlechtsspezifischen Unterschiede zwischen den Skalen des KFS (Dietz et al. 2021) vorlagen. Entsprechend werden die Ergebnisse für Frauen und Männer als gleichbedeutend betrachtet. 


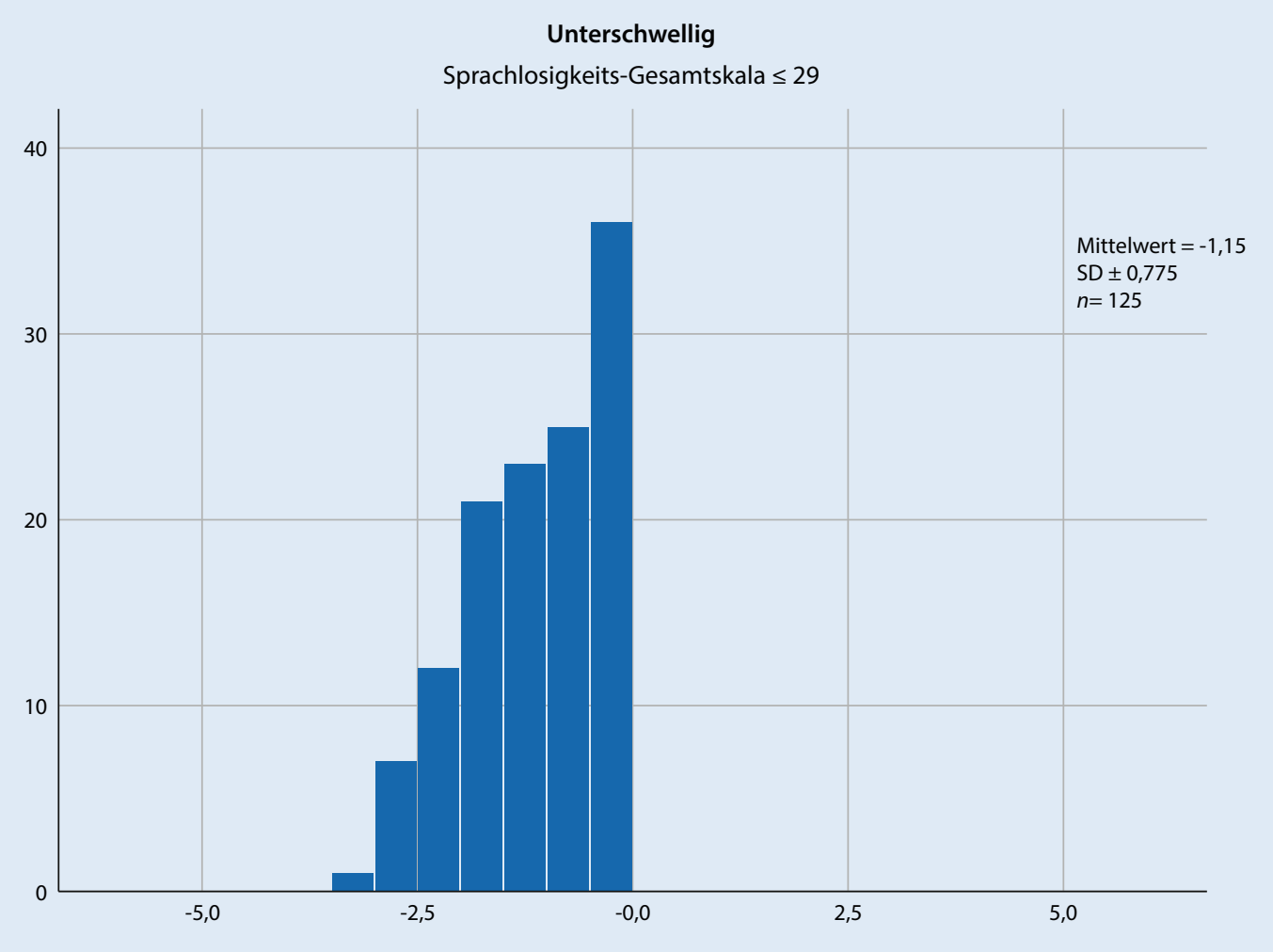

Abb. $3<$ Gruppenzentroiden für unterschwellig sprachlose Personen. SD Standardabweichung

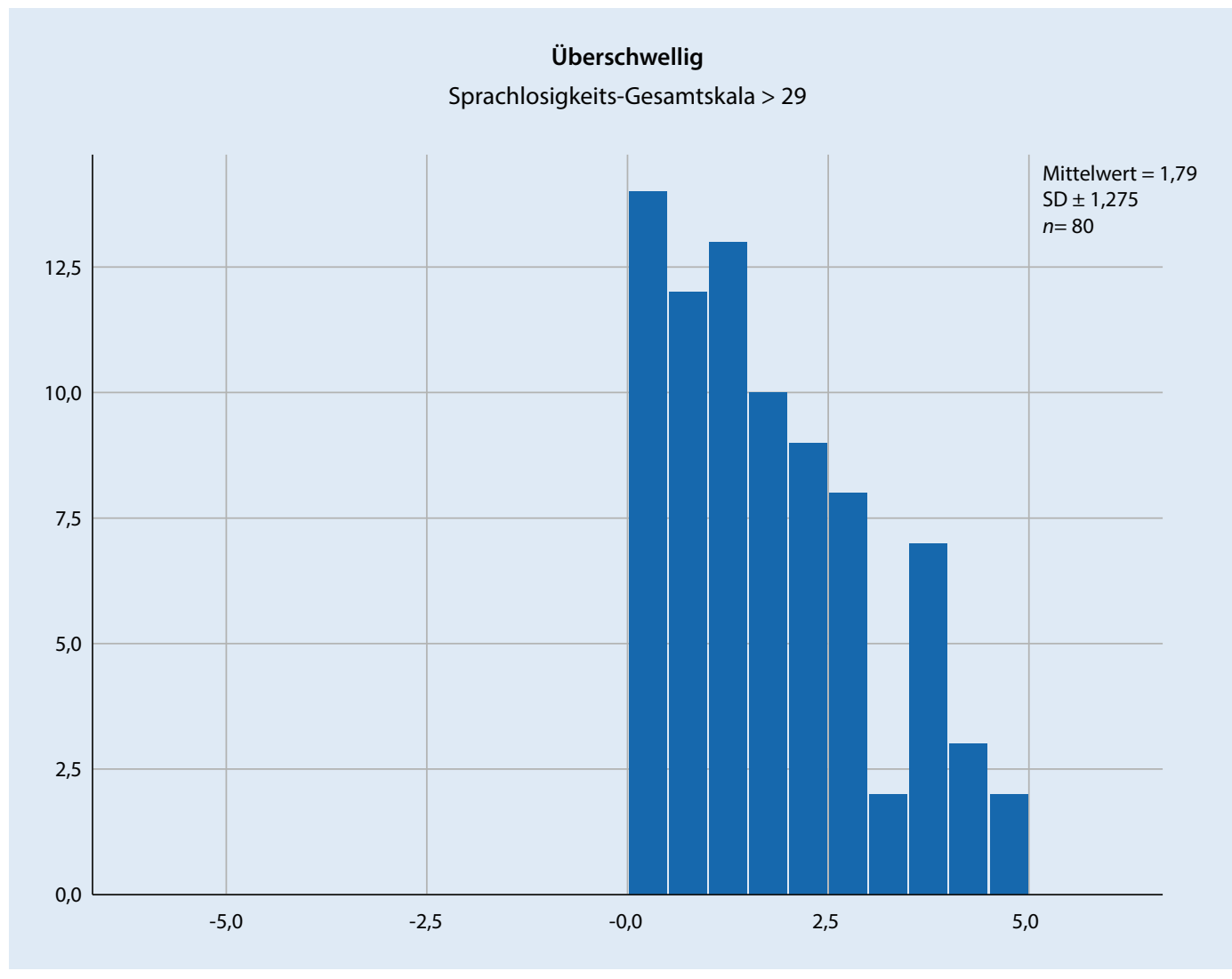

Abb. $4<$ Gruppenzentroiden für überschwellig sprachlose Personen. SD Standardabweichung 
Die Ergebnisse der deskriptiven und inferenzstatistischen Analyse des KFSItem 12 (Dietz et al. 2021) weisen auf hohe prädiktive Eigenschaften des Items hin (•Tab. 1). Sowohl die starken Korrelationen des Items mit den einzelnen (Sub-)Skalen des KFS (Dietz et al. 2021) als auch der Einfluss auf die Sprachlosigkeit (gemessen durch die SprachlosigkeitsGesamtskala; $\bullet$ Tab. 1) bestätigen die normorientierte Testwertinterpretation des Items und auch die Verwendung des Items als externes Kriterium zur Klassifizierung befragter Personen. Die Verwendung des Prozentrang 75 von Item 12 scheint für ein erstes Screening auf Sprachlosigkeit empfehlenswert, um Tendenzen einer stark ausgeprägten Sprachlosigkeit zu erfassen. Eine genaue Erfassung und Differenzierung von Personen sollte jedoch unter Verwendung des KFS-Schwellenwerts erfolgen.

Die zukünftige Auswertung des KFS (Dietz et al. 2021) und ein damit verbundenes Screening sprachloser Personen(-gruppen) können unter Verwendung des KFSSchwellenwerts $>29$ erfolgen. Die Ergebnisse der ROC-Analyse weisen mit einer AUC von 0,863 sowie einer Spezifität von $76 \%$ und einer Sensitivität $79 \%$, verglichen mit den Angaben aus der Literatur, exzellente Ergebnisse auf (Backhaus et al. 2018). Im Vergleich zur Hospital Anxiety and Depression Scale (HADS; Zigmond und Snaith 1983) als Erhebungsinstrument im klinischen Einsatz zeigen sich ähnliche Werte (Singer et al. 2009; $A U C=0,726$; Sensitivität $=76 \%$; Spezifität $=60 \%$ ) für Sensitivität und Spezifität (Hinz und Brähler 2011; Singer et al. 2009). Auch sind die LR $^{-}$und Spezifität des Schwellenwerts geeignet, um den Verdacht einer stark ausgeprägten Sprachlosigkeit zu bestätigen („,rule-in“; Habibzadeh et al. 2016) und die Förderung des "Sprechens" in erster Linie für Personen mit entsprechender Notwendigkeit bereitzustellen (Jäger und Sebastian 2010).

\section{Limitationen}

Bedingt durch die Erhebungsumgebung der Umfrage sind Selektionseffekte bei den teilnehmenden Personen nicht auszuschließen. Der hohe Bildungsschnitt der Stichprobe kann ebenfalls auf die Erhe- bungsumgebung zurückgeführt werden. Bedingt können Verzerrungen in den Ergebnissen und Rückschlüssen somit nicht ausgeschlossen werden. Das Fehlen einer Normalverteilung der Skalen- und Itemwerte kann auf die Stichprobengröße zurückgeführt werden und ist kein Indiz für eine generell fehlende Normalverteilung von Sprachlosigkeit in der Bevölkerung. Es sind weitere Studien mit angepasster Stichprobengröße an Personen der Normalbevölkerung notwendig, um den vorliegenden Schwellenwert zu verifizieren. Auch sollten diese eine möglichst gleiche Geschlechterverteilung vorweisen.

\section{Fazit für die Praxis}

- Schwellenwerte bilden ein zentrales Merkmal des klinischen Einsatzes von Erhebungsinstrumenten, etwa in der Identifikation von Personen mit potenziellen psychischen Komorbiditäten oder der Unterstützung differenzieller Indikationsentscheidungen.

- Unter Verwendung des Schwellenwerts $>29$ der Sprachlosigkeits-Gesamtskala des Kölner Fragebogen zur Sprachlosigkeit (KFS) können Personen mit stark ausgeprägter Sprachlosigkeit identifiziert werden. Die Orientierung an dem (vorläufigen) KFS-Schwellenwert ermöglicht ein Screening auf stark ausgeprägte Sprachlosigkeit und somit die frühzeitige Identifikation betroffener Personen(-gruppen).

\section{Korrespondenzadresse}

\section{Thilo Dietz}

Psychoonkologische Versorgungsforschung, Klinik I für Innere Medizin, Universitätsklinikum Köln

Köln, Deutschland

thilo.dietz@uk-koeln.de

Funding. Das Projekt, in dessen Rahmen die Studie durchgeführt wurde und in dem die in diesem Artikel veröffentlichten Forschungsergebnisse zustande gekommen sind, wurde von der Barbara und Wilfried Mohr-Stiftung gefördert.

Funding. Open Access funding enabled and organized by Projekt DEAL.

\section{Einhaltung ethischer Richtlinien}

Interessenkonflikt. T. Dietz, V. Schiewer, S. Tavenrath, H. Öztürk-Arenz, A. Klein, H. Labouvie, R. Jäger und M. Kusch geben an, dass kein Interessenkonflikt besteht.
Die Studie wurde von der Ethikkommission des Universitätsklinikums überprüft und bewilligt (Aktenzeichen 20-1175).

Open Access. Dieser Artikel wird unter der Creative Commons Namensnennung 4.0 International Lizenz veröffentlicht, welche die Nutzung, Vervielfältigung, Bearbeitung, Verbreitung und Wiedergabe in jeglichem Medium und Format erlaubt, sofern Sie den/die ursprünglichen Autor(en) und die Quelle ordnungsgemäß nennen, einen Link zur Creative Commons Lizenz beifügen und angeben, ob Änderungen vorgenommen wurden.

Die in diesem Artikel enthaltenen Bilder und sonstiges Drittmaterial unterliegen ebenfalls der genannten Creative Commons Lizenz, sofern sich aus der Abbildungslegende nichts anderes ergibt. Sofern das betreffende Material nicht unter der genannten Creative Commons Lizenz steht und die betreffende Handlung nicht nach gesetzlichen Vorschriften erlaubt ist, ist für die oben aufgeführten Weiterverwendungen des Materials die Einwilligung des jeweiligen Rechteinhabers einzuholen.

Weitere Details zur Lizenz entnehmen Sie bitte der Lizenzinformation auf http://creativecommons.org/ licenses/by/4.0/deed.de.

\section{Literatur}

Atkins CGK, Brownell K, Kornelsen J et al (2013) Silos of silence, stress, and suffering: patient and physician experiences of mups and diagnostic uncertainty. AJOBNeurosci 4(3):3-8

Backhaus K, Erichson B, PlinkeWetal (2018) Logistische Regression.In:BackhausK,Erichson B,PlinkeW, al (Hrsg) Multivariate Analysemethoden. Springer Gabler, Berlin, Heidelberg, S267-336

Berger CR (2016) Speechlessness. In: Berger CR, RoloffME, Wilson SRet al (Hrsg) The international encyclopedia of interpersonal communication. John Wiley \& Sons, Hoboken https://doi.org/10. 1002/9781118540190.wbeic216

Bergkvist L, Rossiter JR (2007) The predictive validity of multiple-item versus single-item measures of the same constructs. J Mark Res 44(2):175-184

Canzona MR, Fisher CL, Ledford CJW (2019) Perpetuating the cycle of silence: the intersection of uncertainty and sexual health communication among couples after breast cancer treatment. Support Care Cancer 27(2):659-668

Cohen J (1988) Statistical power analysis for the behavioral sciences, 2. Aufl. Erlbaum, Hillsdale

Dietz T, Tavenrath S, Schiewer V et al. (2021) Kölner Fragebogen zur Sprachlosigkeit, Köln (Unveröffentlicht)

Eskildsen A, Reinholt N, van Bronswijk S et al (2020) Personalized psychotherapy for outpatients with major depression and anxiety disorders: transdiagnostic versus diagnosis-specific group cognitive behavioural therapy. Cognit Ther Res 44:988-1001

Goldhammer F, Hartig J (2020) Testwertinterpretation Testnormen und Testeichung. In: Moosbrugger $\mathrm{H}$, Kelava A (Hrsg) Testtheorie und Fragebogenkonstruktion, 3. Aufl. Springer, Berlin, S171-195

Habibzadeh F, Habibzadeh P, Yadollahie M (2016) On determining the most appropriate test cut-off value: the case of tests with continuous results. 
Biochem Med 26(3):297-307. https://doi.org/ 10.11613/BM.2016.034

Hinz A, Brähler E (2011) Normative values for the hospital anxiety and depression scale (HADS) in the general German population. J Psychosom Res 71(2):74-78

Jäger RS, Sebastian D (2010) Ein Test zur Erfassung von Konzentration und Aufmerksamkeit im Kindergartenalter. Pearson, Frankfurt

Kelava A, Moosbrugger H (2020) Deskriptivstatistische Itemanalyse und Testwertbestimmung. In: Moosbrugger $\mathrm{H}$, Kelava A (Hrsg) Testtheorie und Fragebogenkonstruktion, 3. Aufl. Springer, Berlin, S143-158

Konradsen H, Kirkevold M, McCallin A et al (2012) Breaking the silence: integration of facial disfigurement after surgical treatment for cancer. Qual Health Res 22(8):1037-1046

Kubinger KD (2003) Gütekriterien. In: Kubinger KD, Jäger RS (Hrsg) Schlüsselbegriffe der Psychologischen Diagnostik. Beltz, Weinheim, S195-204

Kupfer J, Brosig B, Brähler E (2001) TAS-26: Toronto-Alexithymie-Skala-26. Hogrefe, Göttingen (deutsche Version)

Kusch M, Stecker R (2005) Die Psychoonkologische Psychotherapie. Forum Psychother Prax 5(1):15-27. https://doi.org/10.1026/1860-7357.5.1.15

Kusch M, Labouvie H, Hein-Nau B et al (2015) Integrierte Psychoonkologie. Tumordiagn Ther 36(02):99-103

Kusch M, Labouvie H, Hein-Nau B (2013) Klinische Psychoonkologie. Springer, Berlin, Heidelberg

Mazzocco MMM, Myers GF (2003) Complexities in identifying and defining mathematics learning disability in the primary school-age years. Ann Dyslexia 53(1):218-253

Mitchell AJ, Lord K, Symonds P (2012) Which symptoms are indicative of DSMIV depression in cancer settings? An analysis of the diagnostic significance of somatic and non-somatic symptoms. J Affect Disord 138(1-2):137-148

Moosbrugger $\mathrm{H}$, Schermelleh-Engel $\mathrm{K}$, Gäde $\mathrm{JC}$ et al (2020) Testtheorie im Überblick. In: Moosbrugger $\mathrm{H}$, Kelava A (Hrsg) Testtheorie und Fragebogenkonstruktion, 3. Aufl. Springer, Berlin, S251-273

Paoletti P, Ben-Soussan TD (2020) Reflections on inner and outer silence and consciousness without contents according to the sphere model of consciousness. Front Psychol 11:1807. https:// doi.org/10.3389/fpsyg.2020.01807

Perkins NJ, Schisterman EF (2006) The inconsistency of "optimal" cutpoints obtained using two criteria based on the receiver operating characteristic curve. Epidemiol Rev 163(7):670-675

Schiewer V, Dietz T, Tavenrath S et al. (2021) Erfassung des korrelativen und faktoriellen Zusammenhangs von Alexithymie und expressiver Suppression: Eine gemeinsame Grundlage, Köln. https://doi.org/10.1007/s00278-021-00546-x

Singer S, Kuhnt S, Götze H et al (2009) Hospital anxiety and depression scale cutoff scores for cancer patients inacute care. BrJCancer 100(6):908-912

Youden WJ (1950) Index for rating diagnostic tests. Cancer 3(1):32-35

Yovanoff P, Squires J (2006) Determining cutoff scores on a developmental screening measure: Use of receiver operating characteristics and item response theory. J Early Interv 29(1):48-62

Zigmond AS, Snaith RP (1983) The hospital anxiety and depression scale. Acta Psychiatr Scand 67(6):361-370

\section{Cologne questionnaire on speechlessness. Empirical testing of a threshold for clinical use}

Background: The Cologne questionnaire on speechlessness (KFS) is the first validated instrument for the assessment of speechlessness. The empirical testing of the KFS regarding its suitability for a valid distinction between persons with low and high levels of speechlessness is a primary goal for the clinical use of the instrument.

Material and methods: The sample comprised the KFS data from 205 participants in an online survey. Using different inferential statistical methods, the predictive properties of the KFS item 12 were analyzed in order to make it useful for the determination of a threshold when using the KFS. Based on the 75th percentile of KFS item 12, a dichotomization of the sample was performed followed by a receiver operating characteristic (ROC) analysis of the KFS overarching total scale speechlessness.

Results: The results of the ROC analysis (area under the curve, AUC: $0.863 ; p<0.001$ ) revealed an optimal threshold of $>29$ (sensitivity $76 \%$; specificity $79 \%$ ) for the total scale of the KFS using the Youden index.

Discussion: The threshold for the KFS speechlessness total scale makes it possible to differentiate people with low or high levels of speechlessness. In addition, item 12 of the KFS can be used to predict potential speechlessness.

\section{Keywords}

Screening $\cdot$ Threshold $\cdot$ ROC curve $\cdot$ Sensitivity and specificity $\cdot$ Affective symptoms 